\title{
Using Doppler sonography resistive index for the diagnosis of perinatal asphyxia: a multi-centered study
}

\section{Parisa Pishdad}

Shiraz University of Medical Sciences

\section{Fatemeh Yarmahmoodi}

Shiraz University of Medical Sciences

\section{Tannaz Eghbali}

Shiraz University of Medical Sciences

\section{Peyman Arasteh}

Shiraz University of Medical Sciences

Seyyed Mostajab Razavi ( $\nabla$ drrazavi1@gmail.com )

Shiraz University of Medical Sciences

\section{Research article}

Keywords: Asphyxia, Perinatal, Ultrasonography, Doppler, Magnetic resonance imaging, Resistive index

Posted Date: August 2nd, 2020

DOl: https://doi.org/10.21203/rs.3.rs-49582/v1

License: (9) This work is licensed under a Creative Commons Attribution 4.0 International License. Read Full License

Version of Record: A version of this preprint was published at BMC Neurology on March 19th, 2022. See the published version at https://doi.org/10.1186/s12883-022-02624-2. 


\section{Abstract}

Background and objective

Inhere we evaluated the efficacy of Doppler sonography (DS) of the anterior cerebral artery (ACA), middle cerebral artery (MCA) and the basilar arteries (BA) based on resistive index (RI) for the diagnosis of asphyxia.

Methods

In this multi-centered cross-sectional study, neonates with clinical diagnosis of asphyxia, were considered for study. During the first 24 hours, neonates underwent DS. MRI was done for each neonate during the first month, after discharge or during hospital admission, after obtaining clinical stability. Staging based on DS was compared with staging based on MRI.

\section{Results}

Overall, 34 patients entered the study. DS of the ACA, MCA, BA all had significant correlation with MRI findings (regarding severity of asphyxia) $(r>0.8$ and $p<0.001)$.

In the receiver-operating-characteristic analysis, ideal cut-off point for diagnoses of asphyxia based on ACA and $B A$ was $\mathrm{RI} \leq 0.62$ [area under the curve $(A U C)=0.957$ and $95 \% \mathrm{Cl}: 0.819-0.997$; sensitivity=95.65; specificity $=100$; positive predictive value $(P P V)=100$; negative predictive value (NPV) $=90.9$ and negative likelihood ratio $(N L R)=0.043$ ]. Regarding $M C A$, similarly, a RI $\leq 0.62$ was ideal for differentiating between normal and asphyxiated neonates (AUC $=0.990$ and $95 \% \mathrm{Cl}$ : 0.873-1; sensitivity $=91.30$; specificity $=100$; $P P V=91.2 ; N P V=100$ and $N L R=0.087)$.

\section{Conclusion}

For evaluating neonates clinically suspected of asphyxia, DS can be used as a first line diagnostic modality and RI of $\leq 0.62$ is an appropriate cut-off for the diagnosis of perinatal asphyxia.

\section{Introduction}

Multiple events during labor and delivery may lead to encephalopathy of the neonate which is generally termed perinatal asphyxia (1). Asphyxia is one of the most common causes of mortality and morbidity among newborns and an estimated one to six from every one thousand full-term births are associated with some degrees of asphyxia (2).

The events that lead to asphyxia cause oxygen deprivation and finally may present as neurological deficit in the neonate. Asphyxia may present with different symptoms including abnormal level of consciousness, difficult respiration, decreased muscle tone and reflexes and etc. (3). 
As asphyxia prolongs, cerebral blood flow is compromised and decreases through systemic hypotension and dysregulation within the cerebral regulatory system. This leads to hypoperfusion and ischemia, which may develop into hypoxic-ischemic encephalopathy and neurological impairment (blindness, hearing loss, cerebral palsy, motor and mental development problems) for the child $(1,4,5)$.

Different modalities have been used to diagnose asphyxia among neonates, among which magnetic resonance imaging (MRI) is considered the gold standard imaging method used in clinical practice (6). Other imaging modalities include ultrasonography, Doppler sonography (DS) of the cerebral arteries, and in some cases computed tomography (CT) (7).

Sonography based imaging, present a non-invasive, portable and safe method of evaluation for patients suspected of asphyxia (8). A reduction in the Resistive index and in increase in the end-diastolic flow velocity have been associated with changes in cerebral blood flow and presents

an effective method for evaluation of asphyxia (9).

In a recent study by Kudreviciene et al., children with asphyxia were studied using DS. They determined a cut-off based on DS index for the prediction of neuro-developmental injuries. Other studied have also studied the prognostic role of DS in children with ischemic brain damage $(4,10,11)$, however at present, data on the diagnostic role of DS in asphyxia remains scarce and DS is not routinely used in the clinical assessment of asphyxia in neonates (9).

Up to this date, no study has yet studied the role of DS in diagnosing children with asphyxia, so in this study we evaluated and compared DS and MRI findings in a sample of neonates suspected of asphyxia, we also determined an optimum cut-off point based on DS (index) of the cerebral arteries to differentiate between neonates with asphyxia and normal neonates.

\section{Patients And Methods}

\section{Study design}

This is a multi-centered cross-sectional study conducted in Namazi, Hafez and Hazrat Zeinab hospitals, affiliated to Shiraz University of Medical Sciences, Shiraz, Iran. During January 2016 to July 2016, neonates who were admitted with a diagnosis of asphyxia based on the Sarnat and Sarnat clinical criteria (12) in the mentioned health care centers, were considered for entry in the study. Neonates born between 37 and 42 weeks who had signs of fetal distress such as abnormal fetal monitoring and presence of meconium in the amnion, with an umbilical cord blood PH of less than 7.1, five minute Apgar score of less than five, were included in the study.

Neonates born earlier than 37 weeks or later than 42 weeks, those who had any congenital disorders (for example patent ductus arteriosus), and those with myocardial dysfunction, were excluded from the study. 
We excluded patients with myocardial dysfunction and congenital abnormalities as some studies have shown these patients to have variable findings regarding DS indexes (13).

\section{Protocol and patient evaluation}

Patients were initially evaluated by a neonatologist and were scored based on the Sarnat and Sarnat scoring system (previously mentioned) as mild, moderate and severe asphyxia. After diagnosis of asphyxia through clinical evaluation by the neonatologist, and after patients were clinically stable, during 6 to 24 hours after birth, each patient underwent DS (Shenzhen Mindray Bio-Medical Electronics Co., China) of the cerebral arteries using a curved probe of $5 \mathrm{MHz}$ and a linear probe of 7.5 MHz. As DS studies are dynamic, DS was done after the first 6 hours (up to 24 hours) of birth in order to obtain stability and more importantly minimize changes in DS findings.

For DS of the ACA, imaging was conducted on bilateral sides on parasagittal planes through the anterior fontanelles. Parameters related to the ACA were measured using a branch of the anterior artery, which were anterior to the corpus callosum. For the MCA, imaging was conducted on bilateral sides by both temporal bones between the eye socket and the ear above the zygoma on axial planes. For the BA, imaging was obtained in the sagittal planes located just before the pons. Examination of the neonates was performed during sleep.

Magnetic resonance imaging (MRI) was done for each neonate during the first month, after discharge or during hospital admission, when the patients obtained a clinically stable condition. Evaluation of MRI findings was done as followed: limited hyper intense T2 signal in brain cortex and subcortical white matter represented mild degree of hypoxic ischemic encephalopathy. Involvement of basal ganglia and thalamus was considered severe hypoxic ischemic encephalopathy. Moreover, mild to moderate encephalopathy were considered disseminated signal changes of the cortex and subcortical weight matter (14). Accordingly, patients were categorized into three groups of normal, mild to moderate (stage 2) and severe (stage 3 ) regarding asphyxia.

MRI and sonography findings were interpreted by two different radiologists who were both unaware of the clinical staging and the staging done by the other radiologist.

Data including sex, one minute and five minute APGAR scores, type of delivery, birth weight, gestational age, clinical severity of asphyxia, severity of asphyxia based on sonography (based on ACA, MCA and $B A)$, and severity of asphyxia based on MRI findings, were all registered in a data gathering sheet.

We used the Sarnat and Sarnat criteria for clinical diagnosis and classification of severity of asphyxia. This criteria considers six variables for the diagnosis of asphyxia including: alertness, muscle tone, seizure, pupils, respiration pattern, and duration of symptoms (12).

For sonography based staging of asphyxia, the Pourcelots's resistive index (RI) was used to estimate cerebral blood flow status, due to its easiness of use, reproducibility and its independence of the angle of insonation (15). 
Peak systolic velocity (PSV), end diastolic velocity (EDV) and resistive index (RI) of the anterior cerebral arteries (ACA), middle cerebral artery (MCA), and the basilar arteries (BA) were measured and the severity of disease was defined, accordingly. Severity of disease was measured according to the RI, which is calculated as the peak systolic velocity minus the end diastolic velocity divided by the systolic velocity. The RI was measured three consecutive times for each artery and the average was considered the final RI.

RI provides a tool to evaluate the dynamics of cerebral blood flow and cerebral pressure (16). Appropriate cut-off points for classifying patients based on RI as sever, moderate and mild were obtained using previous literature (17). According to the mentioned study a cut-off of $\mathrm{RI} \leq 0.57$ was defined as severe asphyxia, moreover according to our final results for the diagnosis of asphyxia (using our own obtained cut-off point for the diagnosis of asphyxia based on RI) the rest of the cut-offs were categorized accordingly. According to our results and that of previous literature (as mentioned before), severe asphyxia was considered as $\mathrm{RI} \leq 0.57$, moderate as $\mathrm{RI}=0.58-0.62$, mild was considered as $\mathrm{RI}=0.63-$ 0.67 , and normal DS was considered as $\mathrm{RI}=0.68-0.72$.

In the end, staging based on sonography was compared with the staging based on MRI findings.

All patients were hospitalized in the neonatal intensive care units and received related intensive care according to standard protocols, therefor factors such as thermoregulation and vasogenic edema which may have affected RI were controlled and were similar for all the patients.

\section{Sample size calculation}

In order to determine a cut-off point for DS, considering a sensitivity of $99 \%$ and a specificity of $67 \%$ and an accuracy of $99 \%$ for a positive likelihood ratio of more than 1.8 , a sample size of 30 individuals was required.

\section{Statistical analysis}

Data was analyzed using the Statistical Package for Social Sciences software (SPSS Inc., Chicago, IL, USA) for windows, version 16.

In order to evaluate the linear correlation between DS findings of the ACA, MCA and BA with MRI, the Spearman's correlation test was used.

To determine the ideal cut-off point regarding RI of the three arteries, in discriminating between normal neonates and those with asphyxia (considering MRI as the gold standard), the receiver operating characteristics (ROC) analysis was used, reporting its area under the curve (AUC), sensitivity, specificity, positive predictive value (PPV), negative predictive value (NPV), positive likelihood ratio (PLR) and negative likelihood ratio (NLR), where appropriate. In addition, a pairwise comparison of ROC curves was performed between the ACA, MCA and BA to determine the difference between the examined arteries.

We calculated the optimum RI cut-off point using the Youden index (18). Using this model, the ideal cutoff point on the ROC curve is considered optimum which has the maximum sensitivity + specificity. Upper 
and lower limits of cut-off points were also determined considering the point on the ROC curve with the highest sensitivity and highest specificity.

Data are presented as means \pm standard deviations (SD) or frequency and percentage, where appropriate.

\section{Results}

Overall, 34 patients entered the study. One patient died before having MRI and was excluded from the study. Patients' baseline characteristics are shown in Table 1. 
Table 1

Patients' baseline characteristics.*

\begin{tabular}{|c|c|}
\hline Variables & Statistics \\
\hline \multicolumn{2}{|l|}{ Sex - no. (\%) } \\
\hline Male & $19(58)$ \\
\hline Female & $14(42)$ \\
\hline \multicolumn{2}{|l|}{ Gestational age - wks } \\
\hline 37 & $10(30.3)$ \\
\hline 38 & $12(36.4)$ \\
\hline 39 & $10(30.3)$ \\
\hline 40 & $1(3)$ \\
\hline \multicolumn{2}{|l|}{ Birth weight - gr } \\
\hline$<2500$ & $2(6.1)$ \\
\hline $2500-4000$ & $30(90.9)$ \\
\hline$>4000$ & 0 \\
\hline \multicolumn{2}{|l|}{ One minute Apgar score } \\
\hline$\leq 5$ & $26(78.8)$ \\
\hline$>5$ & $7(21.2)$ \\
\hline \multicolumn{2}{|l|}{ Five minute Apgar score } \\
\hline$\leq 5$ & $33(100)$ \\
\hline$>5$ & 0 \\
\hline \multicolumn{2}{|l|}{ Birth type } \\
\hline Normal vaginal delivery & $17(51.5)$ \\
\hline Cesarean section & $16(48.5)$ \\
\hline \multicolumn{2}{|l|}{ Clinical severity } \\
\hline Stage 1 & 7 \\
\hline Stage 2 & 16 \\
\hline Stage 3 & 10 \\
\hline \multicolumn{2}{|l|}{ Severity based DS† } \\
\hline Normal & 7 \\
\hline
\end{tabular}




\begin{tabular}{|ll|}
\hline Variables & Statistics \\
\hline Mild & 2 \\
\hline Moderate & 12 \\
\hline Severe & 12 \\
\hline Severity based on MRI & 9 \\
\hline Normal & 14 \\
\hline Stage 2 & 9 \\
\hline Stage 3 & \\
\hline $\begin{array}{l}\text { DS: Doppler sonography } \\
\text { *All data are presented as frequency and percent. } \\
\text { tNormal DS was considered as resistive index }(\mathrm{RI})=0.68-0.72 ; \text { mild was considered as } \mathrm{RI}=0.63- \\
0.67 ; \text { moderate as RI = 0.58-0.62 and severe as RI } \leq 0.57 .\end{array}$ \\
\hline
\end{tabular}

Table 1.

Pearson's correlation showed that, DS of the ACA, MCA, BA all had a significant and strong correlation with MRI findings (regarding severity of asphyxia) $(r>0.8 ; p<0.001)$. DS of the ACA and the BA had the strongest correlation with MRI findings $(r=0.889$ and $p<0.001)$ (Table 2).

Table 2

Correlation between staging based on color Doppler sonography and staging based on MRI of asphyxiated neonates.*

\begin{tabular}{|lllll|}
\hline & DS of ACA & DS of MCA & DS of BA & MRI \\
\hline DS of ACA & 1 & $0.883^{* *}$ & $1 * *$ & $0.889^{* *}$ \\
\hline DS of MCA & $0.883^{* *}$ & 1 & $0.883^{* *}$ & $0.844^{* *}$ \\
\hline DS of BA & $1 * *$ & $0.883^{* *}$ & 1 & $0.889^{* *}$ \\
MRI & $0.889^{* *}$ & $0.844^{* *}$ & $0.889^{* *}$ & 1 \\
\hline $\begin{array}{l}\text { DS: Doppler sonography; ACA: anterior cerebral artery; MCA: middle cerebral artery; BA: basilar artery } \\
* \text { All reported values are correlation coefficients }(r) .\end{array}$ & & \\
$* *$ P $<0.001$ & & & \\
\hline
\end{tabular}

Table 2.

In the ROC analysis, all the arteries were separately evaluated regarding their compatibility with MRI findings. DS of the ACA showed that the ideal cut-off point for diagnosing neonates with asphyxia was at 
a RI of $\leq 0.62$ (AUC $=0.957$ and $95 \%$ Cl: $0.819-0.997$; sensitivity = 95.65; specificity = 100; PPV = 100; $\mathrm{NPV}=90.9$ and NLR $=0.043)$.

Regarding the BA, the exact same results were recorded as the ACA (AUC $=0.957$ and $95 \% \mathrm{Cl}: 0.819-$ 0.997 ; sensitivity $=95.65 ;$ specificity $=100 ; \mathrm{PPV}=100 ; \mathrm{NPV}=90.9$ and NLR $=0.043$ ).

ROC analysis of the MCA showed that, similar to the ACA and BA, a cut-off of $\leq 0.62$ was the ideal cut-off point for differentiating between normal neonates and those with asphyxia (AUC $=0.990$ and $95 \% \mathrm{Cl}$ : 0.873-1; sensitivity $=91.30 ;$ specificity $=100 ;$ PPV $=91.2 ;$ NPV $=100$ and NLR = 0.087) (Table 3) (Fig. 1). 
Table 3

Receiver operating characteristics analysis for the optimum cut-off point for the diagnosis of perinatal asphyxia based on resistive index in Doppler sonography.*

\begin{tabular}{|c|c|c|c|c|c|c|c|}
\hline & AUC & Sensitivity & Specificity & PPV & NPV & PLR & NLR \\
\hline \multicolumn{8}{|l|}{ ACA } \\
\hline$<.46$ & - & $0(0-14.8)$ & $\begin{array}{l}100 \\
(66.4- \\
100)\end{array}$ & & $\begin{array}{l}30.3 \\
(15.4- \\
49)\end{array}$ & & $1(1-1)$ \\
\hline$\leq .62 t$ & $\begin{array}{l}0.957(0.819 \\
\text { to } 0.997)\end{array}$ & $\begin{array}{l}95.65 \\
(78.1- \\
99.9)\end{array}$ & $\begin{array}{l}100 \\
(66.4- \\
100)\end{array}$ & $\begin{array}{l}100 \\
(84.1- \\
100)\end{array}$ & $\begin{array}{l}90.9 \\
(58- \\
99.8)\end{array}$ & & $\begin{array}{l}0.043 \\
(0.006- \\
0.3)\end{array}$ \\
\hline $\begin{array}{l}\leq \\
0.84\end{array}$ & - & $\begin{array}{l}100(85.2- \\
100)\end{array}$ & $\begin{array}{l}0(0- \\
33.6)\end{array}$ & $\begin{array}{l}69.7(51- \\
84.6)\end{array}$ & & & \\
\hline \multicolumn{8}{|l|}{ MCA } \\
\hline $\begin{array}{l}< \\
0.46\end{array}$ & - & $0(0-14.8)$ & $\begin{array}{l}100 \\
(66.4- \\
100)\end{array}$ & & $\begin{array}{l}30.3 \\
(15.4- \\
49)\end{array}$ & & $1(1-1)$ \\
\hline$\leq .62 \ddagger$ & $\begin{array}{l}0.990(0.873 \\
\text { to } 1)\end{array}$ & $\begin{array}{l}91.30 \\
(72.0- \\
98.9)\end{array}$ & $\begin{array}{l}100 \\
(66.4- \\
100)\end{array}$ & $\begin{array}{l}91.2 \\
(72.6- \\
98.8)\end{array}$ & $\begin{array}{l}100 \\
(61.3- \\
100)\end{array}$ & & $\begin{array}{l}0.087 \\
(0.02-0.3)\end{array}$ \\
\hline$\leq$ & - & $\begin{array}{l}100(85.2- \\
100)\end{array}$ & $\begin{array}{l}0(0- \\
33.6)\end{array}$ & $\begin{array}{l}69.7(51- \\
84.6)\end{array}$ & & $\begin{array}{l}1 \\
(1- \\
1)\end{array}$ & $\begin{array}{l}0.96(0.9- \\
1)\end{array}$ \\
\hline \multicolumn{8}{|l|}{ BA } \\
\hline $\begin{array}{l}< \\
0.45\end{array}$ & - & $0(0-14.8)$ & $\begin{array}{l}100 \\
(66.4- \\
100)\end{array}$ & & $\begin{array}{l}30.3 \\
(15.4- \\
49)\end{array}$ & & 1 \\
\hline$\leq$ & $\begin{array}{l}0.957(0.819 \\
\text { to } 0.997)\end{array}$ & $\begin{array}{l}95.65 \\
(78.1- \\
99.9)\end{array}$ & $\begin{array}{l}100 \\
(66.4- \\
100)\end{array}$ & $\begin{array}{l}100 \\
(84.1- \\
100)\end{array}$ & $\begin{array}{l}90.9 \\
(58- \\
99.8)\end{array}$ & & $\begin{array}{l}0.043 \\
(0.006- \\
0.3)\end{array}$ \\
\hline$\leq$ & - & $\begin{array}{l}100(85.2- \\
100)\end{array}$ & $\begin{array}{l}0(0- \\
33.6)\end{array}$ & $\begin{array}{l}69.7(51- \\
84.6)\end{array}$ & & $\begin{array}{l}1 \\
(1- \\
1)\end{array}$ & \\
\hline
\end{tabular}



AUC
Sensitivity
Specificity
PPV
NPV
PLR NLR

AUC: area under curve; PPV: positive predictive value; NPV: negative predictive value; PLR: positive likelihood ratio; NLR: negative likelihood ratio; ACA: anterior cerebral artery; MCA: middle cerebral artery; BA: basilar artery

*All values are presented with their $95 \%$ confidence interval. Upper and lower limits were chosen on the ROC curve considering the highest sensitivity or specificity.

the Youden index score at this cut-off was 0.956.

$\ddagger$ The Youden index score at this cut-off was 0.956 .

$\S$ The Youden index score at this cut-off was 0.913.

Table 3.

Figure 1.

Results of pairwise comparison of ROC curves, showed that the ROC curves between the ACA and BA (which were completely similar $p>0.99)$ did not have a significant difference with that of the MCA $(p=$ 0.39).

\section{Discussion}

In this study we evaluated DS of the ACA, MCA and BA among neonates clinically diagnosed with asphyxia and compared it with MRI findings, which is the gold standard tool for the diagnosis of neonatal asphyxia, furthermore we defined a cut-off point for RI at which DS is able to detect neonatal asphyxia. We found that a $\mathrm{RI} \leq 0.62$ in DS, is the optimum cut-off point for the diagnosis of perinatal asphyxia with an accuracy of $95 \%$ (for the ACA and the BA) and $99 \%$ (for the MCA).

To the best of the author's knowledge this is the first study to compare DS findings with MRI findings in order to determine a cut-off point based on RI for the diagnosis of perinatal asphyxia. Up to this date, studies evaluating DS in asphyxia have been mostly old, have focused on prognosis (long term neurodevelopmental outcome) and are less applicable in clinical practice for discriminating between neonates with asphyxia and normal neonates.

In a study by Kudreviciene et al. in 2014 (11), one year prognosis was evaluated among neonates with hypoxic brain injuries and normal neonates, using ultrasonography (US) and DS. They found that neonates with a RI $\leq 0.55$ in the ACA on days $1-5$ of birth, had significantly higher watershed border zone injury, thalamus, basal ganglia and cerebellar injuries.

Ilves et al. (19) also evaluated cerebral blood flow among infants with asphyxia in order to predict long term outcomes. They found that when evaluating cerebral arteries during the first 24 hours of birth (similar to that of our study), infants with poor outcome or those with severe hypoxic ischemic encephalopathy had a lower RI in the BA, MCA and carotid arteries compared to a control group. 
In another study (20), US indices were measured among 212 patients, in order to determine the validity of US in predicting three year adverse outcome among children with encephalopathy. In this study, US findings were evaluated during 24 and 72 hours after birth. They found that among 39 neonates who had US during the first 24 hours of life and had their RI measured, those with a RI $<0.56$ had a 23.6 time higher chance (95\% Cl: $2.6-217.5$, Sensitivity $=53 \%$, specificity $=95 \%, \mathrm{PPV}=90 \%$ and NPV $=72 \%$ ) of developing adverse outcomes.

A higher threshold for RI $(<0.60)$ in the ACA and the MCA was documented in an older study by Stark et al. (compared to similar studies) to be associated with poor five year clinical outcomes among 16 term asphyxiated neonates (21).

Some older studies have also evaluated the relationship between neurodevelopment of neonates and cerebral blood flow indices, and have mostly documented similar findings to the previously mentioned studies regarding decreased cerebral blood flow and its association with adverse long term outcomes (17, $22,23)$.

In here we found that a cut-off of $\leq 0.62$ for RI is a diagnostic threshold for asphyxia among full-term neonates. This is in coherence with previous literature, furthermore to date, studies that have evaluated RI cut-off points have all been based on clinical outcomes and prognosis among asphyxiated neonates and have all documented lower cut-off thresholds than that documented in our study. Perhaps a cause for the lower cut-off points documented in different studies from that documented in our study, is that not all asphyxiated children necessarily show poor long term neurodevelopmental outcomes (for example some milder forms of the disease) and so when evaluating cut-off points based on prognosis (as in the mentioned studies) a lower threshold is documented.

One of the main causes for the difference documented between studies regarding RI cut-off points, relates to the timing of the initial DS. A study documented no significant difference in RI when performing DS during specific hours of birth between children with asphyxia and normal infants, however this difference was mostly significant during the 24 hours of birth between these two groups (19), thus pointing the importance of timing of DS for comparison among studies.

For facilities where MRI (as the gold standard diagnostic modality) is too expensive or is unavailable in perinatal care centers, based on our results a RI of $\leq 0.62$ in DS performed on the first day of birth can be considered an appropriate diagnostic cut-off point for neonates suspected of asphyxia. This provides a tool for easy and less costly diagnosis of asphyxia and provides clinicians with an index with high precision for diagnosis of the condition.

Our study did have some limitations. Our cut-off points were based on DS performed on the first day of birth and for those neonates for whom DS is not performed on the first day, the reported cut-off points are not applicable. Another factor which is present in almost every study evaluating sonography findings, is the operator dependency and the subjective nature of sonography. This shows that for sonography to be considered an efficient tool for the evaluation of asphyxia, there is need for expert staff members to 
perform and evaluate sonography findings. Factors such as hypothermia and vasogenic edema are known to affect RI (24-26), considering that all our patients received appropriate ICU care and were continuously monitored none of these caused any issue or bias in our measurements.

\section{Conclusion}

For evaluating neonates clinically suspected of asphyxia, DS can be used as a first line diagnostic modality for the diagnosis of asphyxia. A RI of $\leq 0.62$ is an appropriate cut-off to differentiate between normal and asphyxiated neonates with excellent accuracy.

\section{Abbreviations}

Doppler sonography (DS), anterior cerebral artery (ACA), middle cerebral artery (MCA), basilar arteries $(B A)$, resistive index $(\mathrm{RI})$, magnetic resonance imaging (MRI), computed tomography $(\mathrm{CT})$, peak systolic velocity (PSV), end diastolic velocity (EDV), resistive index (RI), area under the curve (AUC), positive predictive value (PPV), negative predictive value (NPV), positive likelihood ratio (PLR) and negative likelihood ratio (NLR).

\section{Declarations}

\section{Ethics approval and consent to participate}

The study protocol was approved by the Institutional Review Board of Shiraz University of Medical Sciences. All patients (legal guardians) gave their informed and written consent to enter the study.

\section{Consent for publication}

Patients (legal guardians) gave their written and informed consent for the publication of data.

\section{Availability of data and material}

Authors and institution may request the data from the study by directly contacting the corresponding author.

\section{Competing interests}

Authors have no competing interest to declare regarding the manuscript.

\section{Funding}

The study was funded by Shiraz University of Medical Sciences (Grant \#10443-48-01-94). 


\section{Authors' contributions}

PP, FY and SMR aided in study conceptualization, design, gathering of data and critical revision of final paper. TE and PA aided in interpretation of results, preparation of final draft of manuscript. All authors approved the final manuscript.

\section{Acknowledgement}

Authors would like to thank the patient for their kind participation and cooperation in our study.

\section{References}

1. Herrera CA, Silver RM. Perinatal Asphyxia from the Obstetric Standpoint: Diagnosis and Interventions. Clinics in perinatology. 2016;43(3):423-38.

2. de Haan M, Wyatt JS, Roth S, Vargha-Khadem F, Gadian D, Mishkin M. Brain and cognitivebehavioural development after asphyxia at term birth. Dev Sci. 2006;9(4):350-8.

3. Executive summary: Neonatal encephalopathy and neurologic outcome, second edition. Report of the American College of Obstetricians and Gynecologists' Task Force on Neonatal Encephalopathy. Obstetrics and gynecology. 2014;123(4):896-901.

4. Cassia GS, Faingold R, Bernard C, Sant'Anna GM. Neonatal hypoxic-ischemic injury: sonography and dynamic color Doppler sonography perfusion of the brain and abdomen with pathologic correlation. AJR American journal of roentgenology. 2012;199(6):W743-52.

5. Jacobs S, Hunt R, Tarnow-Mordi W, Inder T, Davis P. Cooling for newborns with hypoxic ischaemic encephalopathy. The Cochrane database of systematic reviews. 2007(4):Cd003311.

6. Epelman M, Daneman A, Kellenberger CJ, Aziz A, Konen O, Moineddin R, et al. Neonatal encephalopathy: a prospective comparison of head US and MRI. Pediatric radiology. 2010;40(10):1640-50.

7. Kudreviciene A, Lukosevicius S, Laurynaitiene J, Marmiene V, Tameliene R, Basevicius A. Ultrasonography and magnetic resonance imaging of the brain in hypoxic full-term newborns. Medicina. 2013;49(1):42-9.

8. Han BH, Song MJ, Lee KS, Kim YH, Ko SY, Jung G, et al. Superficial Echogenic Lesions Detected on Neonatal Cranial Sonography: Possible Indicators of Severe Birth Injury. Journal of ultrasound in medicine: official journal of the American Institute of Ultrasound in Medicine. 2016;35(3):477-84.

9. Antonucci R, Porcella A, Pilloni MD. Perinatal asphyxia in the term newborn. Journal of Pediatric Neonatal Individualized Medicine (JPNIM). 2014;3(2):e030269.

10. Julkunen MK, Uotila J, Eriksson K, Janas M, Luukkaala T, Tammela O. Obstetric parameters and Doppler findings in cerebral circulation as predictors of 1 year neurodevelopmental outcome in 
asphyxiated infants. Journal of perinatology: official journal of the California Perinatal Association. 2012;32(8):631-8.

11. Kudreviciene A, Basevicius A, Lukosevicius S, Laurynaitiene J, Marmiene V, Nedzelskiene I, et al. The value of ultrasonography and Doppler sonography in prognosticating long-term outcomes among full-term newborns with perinatal asphyxia. Medicina. 2014;50(2):100-10.

12. Sarnat HB, Sarnat MS. Neonatal encephalopathy following fetal distress. A clinical and electroencephalographic study. Arch Neurol. 1976;33(10):696-705.

13. Daneman A, Epelman M, Blaser S, Jarrin JR. Imaging of the brain in full-term neonates: does sonography still play a role? Pediatric radiology. 2006;36(7):636-46.

14. Chao CP, Zaleski CG, Patton AC. Neonatal hypoxic-ischemic encephalopathy: multimodality imaging findings. Radiographics: a review publication of the Radiological Society of North America Inc. 2006;26(suppl_1):159-S72.

15. Archer LN, Levene MI, Evans DH. Cerebral artery Doppler ultrasonography for prediction of outcome after perinatal asphyxia. Lancet. 1986;2(8516):1116-8.

16. Liao HT, Hung KL. Anterior cerebral artery Doppler ultrasonography for prediction of outcome after perinatal asphyxia. Zhonghua Minguo xiao er ke yi xue hui za zhi [Journal] Zhonghua Minguo xiao er ke yi xue hui. 1997;38(3):208-12.

17. Nishimaki S, Iwasaki S, Minamisawa S, Seki K, Yokota S. Blood flow velocities in the anterior cerebral artery and basilar artery in asphyxiated infants. Journal of ultrasound in medicine: official journal of the American Institute of Ultrasound in Medicine. 2008;27(6):955-60.

18. Youden WJ. Index for rating diagnostic tests. Cancer. 1950;3(1):32-5.

19. Ilves P, Lintrop M, Metsvaht T, Vaher U, Talvik T. Cerebral blood-flow velocities in predicting outcome of asphyxiated newborn infants. Acta paediatrica (Oslo, Norway: 1992). 2004;93(4):523-8.

20. Jongeling BR, Badawi N, Kurinczuk JJ, Thonell S, Watson L, Dixon G, et al. Cranial ultrasound as a predictor of outcome in term newborn encephalopathy. Pediatr Neurol. 2002;26(1):37-42.

21. Stark JE, Seibert JJ. Cerebral artery Doppler ultrasonography for prediction of outcome after perinatal asphyxia. Journal of ultrasound in medicine: official journal of the American Institute of Ultrasound in Medicine. 1994;13(8):595-600.

22. Kirimi E, Tuncer O, Atas B, Sakarya ME, Ceylan A. Clinical value of color Doppler ultrasonography measurements of full-term newborns with perinatal asphyxia and hypoxic ischemic encephalopathy in the first 12 hours of life and long-term prognosis. Tohoku J Exp Med. 2002;197(1):27-33.

23. Fukuda $S$, Kato T, Kuwabara $S$, Kato I, Futamura M, Togari $H$. The ratio of flow velocities in the middle cerebral and internal carotid arteries for the prediction of cerebral palsy in term neonates. Journal of ultrasound in medicine: official journal of the American Institute of Ultrasound in Medicine. 2005;24(2):149-53.

24. Elstad M, Whitelaw A, Thoresen M. Cerebral Resistance Index is less predictive in hypothermic encephalopathic newborns. Acta paediatrica (Oslo, Norway: 1992). 2011;100(10):1344-9. 
25. Pinto PS, Tekes A, Singhi S, Northington FJ, Parkinson C, Huisman TAGM. White-gray matter echogenicity ratio and resistive index: sonographic bedside markers of cerebral hypoxic-ischemic injury/edema? Journal of perinatology: official journal of the California Perinatal Association. 2012;32(6):448-53.

26. Don S, Kopecky K, Filo R, Leapman S, Thomalla J, Jones J, et al. Duplex Doppler US of renal allografts: causes of elevated resistive index. Radiology. 1989;171(3):709-12.

\section{Figures}
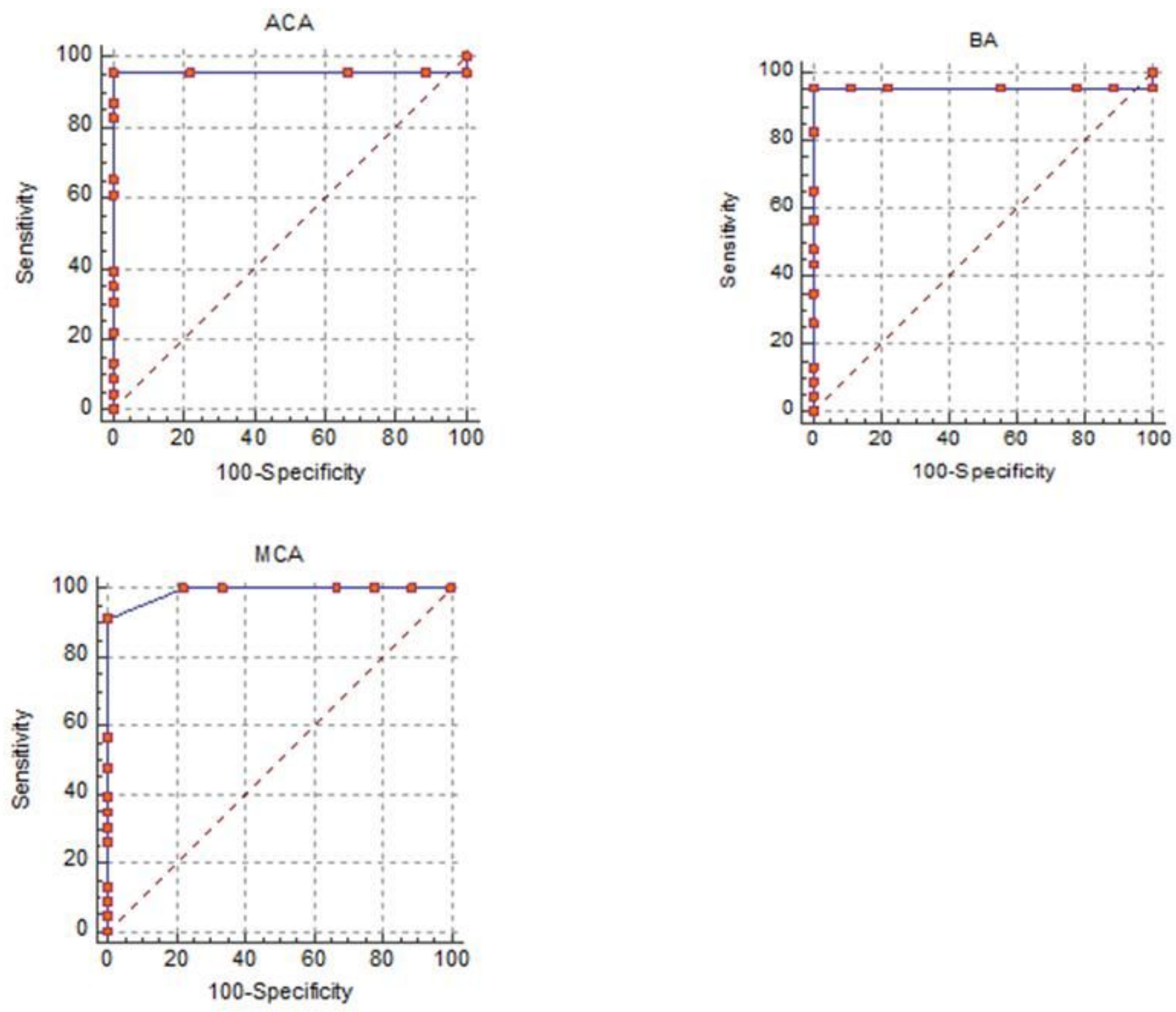

\section{Figure 1}

ROC curves of the ACA, MCA and BA for defining the ideal cut-off point based on resistive index for the diagnosis of perinatal asphyxia. 